\title{
The content of ATP Synthase in Cerebral Ischemia of Varying Severity Comparative Analysis
}

\author{
Bon Elizaveta I. ${ }^{*}$, Maksimovich Natalia E. ${ }^{2}$, Karnyushko Olga A. ${ }^{3}$, Zimatkin Sergey M. ${ }^{4}$ and Lychkovskaya Maria $\mathrm{A}^{5}$
}

${ }^{1}$ Candidate of Biological Sciences, Associate Professor of the Department of Pathological Physiology named after D.A. Maslakova Grodno State Medical University, Repablic of Belarus.

${ }^{2}$ Doctor of Medical Sciences, Professor, Head of the Department of Pathological Physiology named after D.A. Maslakova Grodno State University, Republic of Belarus.

${ }^{3}$ Candidate of Biological Sciences, Associate Professor of the Department of Histology, Cytology and Embryology, Grodno State University, Republic of Belarus.

${ }^{4}$ Doctor of Biological Sciences, Professor, Head of the Department of Histology, Cytology and Embryology Grodno State University, Republic of Belarus..

${ }^{5}$ A fourth-year student of the group number 7 of faculty of Pediatrics Medicine Grodno State Medical University, Republic of Belarus.

*Corresponding Author: Lizaveta I. Bon, Candidate of biological science, Associate professor of pathophysiology department named D.A. Maslakov, Grodno State Medical University, Belarus.

Received date: November 16, 2021; Accepted date: December 02, 2021; Published date: January 04, 2022

Citation: Bon Elizaveta I., Maksimovich Natalia E., Karnyushko Olga A., Zimatkin Sergey M. and Lychkovskaya Maria A (2022) The content of ATP Synthase in Cerebral Ischemia of Varying Severity Comparative Analysis. J, Biotechnology and Bioprocessing 3(1); DOI: 10.31579/27662314/064

Copyright: () 2022, Lizaveta I. Bon, This is an open access article distributed under the Creative Commons Attribution License, which permits unrestricted use, distribution, and reproduction in any medium, provided the original work is properly cited.

\section{Abstract}

Objective. Evaluation of changes in the content of ATP synthase in the parietal cortex and hippocampus of the brain of rats with ischemia of varying severity in a comparative aspect.

Methods. The experiments were performed on 88 male outbred white rats weighing $260 \pm 20 \mathrm{~g}$. Brain ischemia was modeled under conditions of intravenous thiopental anesthesia $(40-50 \mathrm{mg} / \mathrm{kg})$. Total cerebral ischemia was modeled by decapitation of animals. The brain sampling was carried out 1 hour and 24 hours after decapitation - to study tissue respiration of mitochondria, as well as 1 hour later to determine the content of ATP synthase. Subtotal cerebral ischemia was modeled by simultaneous ligation of both common carotid arteries. The material was taken after 1 hour to determine the content of ATP synthase. Stepwise subtotal cerebral ischemia was performed by sequential ligation of both common carotid arteries with an interval of 7 days. The sampling was carried out 1 hour after ligation of the second common carotid artery in each of the subgroups. Partial cerebral ischemia was modeled by ligation of one common carotid artery on the right. The sampling was carried out 1 hour after the operation.

Determination of the content of ATP synthase was carried out by immunohistochemical method using monoclonal antibodies. For this purpose, after decapitation, the brain was quickly removed from the rats, pieces of the cerebral cortex were fixed in zinc-ethanol-formaldehyde at $+4{ }^{\circ} \mathrm{C}$ (overnight), then embedded sin sparaffin.

Results. In the group of stepwise subtotal cerebral ischemia, the smallest decrease in the content of ATP synthase was observed in the 1 st subgroup with an interval between dressings of 7 days, while the greatest decrease in the content of the enzyme was noted in the 3rd subgroup with the minimum interval between the dressings of the common carotid artery ( 1 day). Modeling of more severe types of ischemic damage led to pronounced morphological changes in neurons in the parietal cortex and hippocampus of the rat brain - a decrease in their size, deformation of the perikarya, an increase in the degree of neuronal chromatophilia with their simultaneous wrinkling and subsequent death. These disorders were most pronounced in the 3rd subgroup of stepwise subtotal cerebral ischemia with the shortest interval between dressings, which was 1 day, and in the group of total cerebral ischemia.

Conclusion. Thus, the most pronounced decrease in the content of ATP synthase was observed in the groups of total cerebral ischemia, subtotal cerebral ischemia and in the 3rd subgroup of stepwise subtotal cerebral ischemia, with a minimal time interval 
between the ligation of the common carotid artery. In stepwise subtotal cerebral ischemia with an interval between ligation of the common carotid artery of 7 days, the suppression of the ATP synthase content was not so significant.

Key words: ATP-synthase; cerebral ischemia; neurons

\section{Introduction}

In cerebral ischemia (CI), a chain of pathogenetic disorders develops in its structures, among which one of the leading is energy deficiency, which leads to the development of cellular pathology [1, 2]. The work of enzymes, including sodium-potassium ATPase, is disrupted, leading to an imbalance of ions and cerebral edema $[3,4,5,6]$.

A number of molecular markers of the energy activity of mitochondria are known, among which one of the main markers is ATP synthase - an integral protein of the inner mitochondrial membrane that carries out the reaction of ATP formation from ADP [7, 8]. Mitochondrial ATP synthase plays an important role in the differentiation of stem cells, promotes the formation of mitochondrial cristae by dimerization and specific regulation $[9,10,11]$. The enzyme belongs to the alpha / beta ATP synthase family. Consists of two structural domains (F1 - extramembrane catalyst and F0 - membrane proton channel), connected by a central rod consisting of $\gamma$, $\delta$ and $\varepsilon$ subunits $[12,13,14,15]$, and together with the oligomer of the membrane subunit representing the rotary domain of the enzyme $[1,4,7,16,17]$.

Elucidation of the mechanisms of the development of energy deficiency in ischemic damage of varying severity is advisable for detailing the pathogenesis, the ratio of damage and compensation processes in СШ.

The aim of the study was to assess changes in the content of ATP synthase in the parietal cortex and hippocampus of the brain of rats with ischemia of varying severity in a comparative aspect.

For this purpose, the changes in the content of ATP synthase in rats with partial, subtotal, stepwise subtotal and total CI were studied [3].

\section{Methods}

The experiments were carried out on 88 male outbred white rats weighing $260 \pm 20 \mathrm{~g}$ in compliance with the Directive of the European Parliament and of the Council No. 2010/63/EU of 22.09.2010 on the protection of animals used for scientific purposes.

CI was modeled under conditions of intravenous thiopental anesthesia (40-50 mg/kg). Total cerebral ischemia (TCI) was modeled by decapitation of animals. The brain sampling was carried out 1 hour and 24 hours after decapitation to study tissue respiration of mitochondria, and also after 1 hour to determine the content of ATP synthase.

Subtotal cerebral ischemia (SCI) was modeled by simultaneous ligation of both common carotid arteries (CCA). The material was taken after 1 hour to determine the content of ATP synthase.

Graduated subtotal CI (GSCI) was performed by sequential ligation of both CCA with an interval of 7 days (subgroup 1), 3 days (subgroup 2), or 1 day (subgroup 3). The sampling of material was carried out 1 hour after ligation of the second CCA in each of the subgroups. Partial cerebral ischemia (PCI) was modeled by ligating one CCA on the right. The sampling of material was carried out 1 hour after the operation $[3,18]$.

Determination of the content of ATP synthase was carried out by immunohistochemical method using monoclonal antibodies. For this purpose, after decapitation, the brain was quickly removed from the rats, pieces of the cerebral cortex were fixed in zinc-ethanol-formaldehyde at $+4^{\circ} \mathrm{C}$ (overnight), then embedded in paraffin.

Paraffin sections with a thickness of $5 \mu \mathrm{m}$ were prepared using a microtome and mounted on glass slides. The preparations were processed according to the protocol of immunocytochemical reaction for light microscopy, excluding the procedure of thermal unmasking of antigens. To determine the immunoreactivity of the molecular marker of mitochondria ATP synthase (complex V that forms ATP from ADP), primary monoclonal mouse antibodies (Anti-ATP5A antibody, Abcam, UK, ab. 14748) were used at a dilution of $1: 2400$ at $+4^{\circ} \mathrm{C}$, with an exposure of $20 \mathrm{~h}$ in a humid chamber. To detect bound primary antibodies, an EXPOSE Mouse and Rabbit specific HRP/DAB detection IHC kit Abcam (Great Britain, ab. 80436) was used. The immunoreactivity of ATP synthase was studied in the cytoplasm of neurons of the fifth layer of the parietal cortex and neurons of the CA1 field of the hippocampus in immunohistochemical preparations based on the optical density of the chromogen sediment using an Axioscop 2 plus microscope (Zeiss, Germany), a digital video camera (Leica DFC 320, Germany) and an image analysis program ImageWarp (Bitflow, USA).

To prevent systematic measurement errors, brain samples from the compared control and experimental groups of animals were studied under the same conditions.

As a result of research, quantitative continuous data were obtained. In connection with the use of small samples with an abnormal distribution in the experiment, the processing was carried out by the methods of nonparametric statistics using the licensed computer program Statistica 10.0 for Windows (StatSoft, Inc., USA). Data are presented as Me (LQ; UQ), where Me is the median, LQ is the value of the lower quartile; UQ is the upper quartile value. Differences between groups were considered significant at $\mathrm{p}<0.05$ (Kruskell-Wallis test with Bonferoni's correction) [19].

\section{Results}

In TCI, a decrease in the content of ATP synthase, which characterizes the state of the V-complex of the electron transport chain, was noted in comparison with its level in the control group - by $74(66 ; 86) \%$ in the parietal cortex, $\mathrm{p}<0.05$ and by $70(54 ; 81) \%$ - in the hippocampus, $\mathrm{p}<0.05$ (table 1, figures 1,2). At the same time, there were no differences in the content of the enzyme in the parietal cortex and hippocampus ( $p>0.05)$. 


\begin{tabular}{|c|c|c|c|}
\hline \multirow{2}{*}{\multicolumn{2}{|c|}{ Groups }} & \multicolumn{2}{|c|}{ ATP-synthase content/optical density unit } \\
\hline & & parietal cortex & hippocampus \\
\hline \multicolumn{2}{|c|}{ Control II } & $0,198(0,193 ; 0,209)$ & $0,181(0,176 ; 0,183)$ \\
\hline \multicolumn{2}{|c|}{$\mathrm{TCI}$} & $0,047(0,046 ; 0,055)^{*}$ & $0,066(0,043 ; 0,074)^{*}$ \\
\hline \multicolumn{2}{|l|}{ SCI } & $0,109(0,103 ; 0,121) * \#$ & $0,107(0,104 ; 0,115) * \#$ \\
\hline \multicolumn{2}{|l|}{ PCI } & $0,158(0,147 ; 0,162)^{*} \#+$ & $0,165(0,163 ; 0,170)^{*} \#+$ \\
\hline \multirow[b]{3}{*}{ 它 } & \begin{tabular}{|l|}
1 sg \\
(7 days)
\end{tabular} & $0,157(0,151 ; 0,159)^{*}$ & $0,166(0,164 ; 0,171)^{*}$ \\
\hline & $\begin{array}{l}2 \mathrm{sg} \\
\text { (3 days) }\end{array}$ & $0,129(0,118 ; 0,134) * \alpha$ & $0,132(0,131 ; 0,135) * \alpha$ \\
\hline & $\begin{array}{l}3 \mathrm{sg} \\
\text { (1 days) }\end{array}$ & $0,117(0,109 ; 0,124) * \alpha$ & $0,121(0,113 ; 0,122)^{*} \alpha \beta$ \\
\hline
\end{tabular}

Note: $*$ - $p<0.05$ compared with the "control" group, \# - $\mathrm{p}<0.05$ compared with the "TCI" group, $+-\mathrm{p}<0.05$ compared with the "SCI" group, $\alpha-\mathrm{p}<0.05$ compared with, compared with the 1st subgroup of SCI,

$\beta$ - $\mathrm{p}<0.05$ in comparison with the 2 nd subgroup of GSCI. TCI - total cerebral ischemia, SCI - subtotal cerebral ischemia, PCI - partial cerebral ischemia, SSCI - stepwise subtotal cerebral ischemia, sg - subgroup

Table 1. The content of ATP synthase in the cytoplasm of neurons in the parietal cortex and field CA1 of the hippocampus of rats with total, subtotal and partial cerebralsischemia,sMes(LQ;sUQ)

Compared with the value in the "control", in the SCI group, the content of ATP synthase decreased by $45(38 ; 53) \%$ - in the parietal cortex (p $<0.05)$ and by $39(31 ; 46) \%$ - in the hippocampus $(\mathrm{p}<0.05)$.

Compared to the level of the value in the "TCI" group, the content of ATP synthase in rats with SCI was higher in the parietal cortex by 57 (46; $61) \%, \mathrm{p}<0.05$ and by $38(32 ; 43) \%$ - in the hippocampus, $\mathrm{p}<0.05$.

The decrease in the content of ATP synthase in rats with SIGM was less pronounced than in rats with TCI - by $29 \%$ in the parietal cortex $(\mathrm{p}<0.05)$ and by $39 \%$ in the hippocampus ( $\mathrm{p}<0.05)$.

With PCI, there was a decrease in the content of ATP synthase, compared with the value in the control group - in the parietal cortex by $21(13 ; 32) \%$, $\mathrm{p}<0.05$, in the hippocampus by $8(4 ; 13) \%, \mathrm{p}<0,05$.

At the same time, compared with the value in the "TIGM" group, the content of ATP synthase in the cytoplasm of neurons was $70(66 ; 85) \%$ higher in the parietal cortex $(\mathrm{p}<0.05)$ and by $58(41 ; 76) \%$ - in the hippocampus ( $\mathrm{p}<0.05$ ), and compared with the value in the "SCI" group - by $28(19 ; 38) \%, \mathrm{p}<0.05$ and $35(27 ; 42) \%$, p <0.05, respectively.

A decrease in the ATP synthase content in the parietal cortex and hippocampus with PCI is less pronounced than with SIGM by $24 \%$ (p $<0.05)$ and $31 \%(\mathrm{p}<0.05)$, respectively, and than with TCI - by $53 \%$ (p $<0.05)$ and $62 \%(\mathrm{p}<0.05)$, respectively.

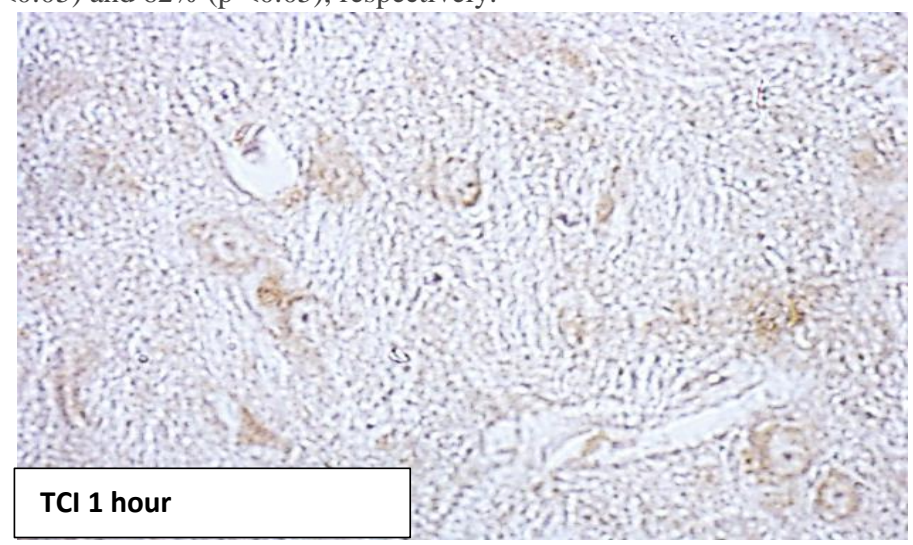

Compared to the level of ATP synthase in the control group, in the 1st subgroup of SCI (the interval between dressings is 7 days), there was a decrease in the content of ATP synthase by $23(12 ; 37) \%$ in the parietal cortex $(\mathrm{p}<0,05)$ and by $7(3 ; 12) \%$ - in the hippocampus $(\mathrm{p}<0.05)$, in the 2nd subgroup of GSCI (the interval between dressings is 3 days), the content of ATP synthase decreased to a greater extent, amounting to 37 $(27 ; 44) \%$ - in the parietal cortex $(\mathrm{p}<0.05)$ and $26(20 ; 34) \%$ - in the hippocampus ( $\mathrm{p}<0.05$ ), and in the 3rd subgroup (the interval between dressings 1 day) there was the most significant decrease enzyme content - by $42(35 ; 52) \%$. p <0.05 and by $33(27 ; 42) \%$, p <0.05, respectively (table. 2).

In the 3rd subgroup of GSCI (the interval between CCA dressings was 1 day), the enzyme content was less, compared with the 1st subgroup, by $27(22 ; 32) \%$ - in the parietal cortex $(\mathrm{p}<0.05)$ and by $18(11 ; 23) \%$ - in the hippocampus ( $\mathrm{p}<0.05$ ), and compared with the 2nd subgroup of GSCI - by $8(3 ; 15) \%$ in the hippocampus $(\mathrm{p}<0.05)$, while in the parietal cortex there were no differences was noted $(p>0.05)$.

Thus, in the "GSCI" group, the smallest decrease in the content of ATP synthase was observed in the 1st subgroup of GSCI with an interval between dressings of 7 days, while the greatest decrease in the enzyme content was noted in the 3 rd subgroup with a minimum interval between CCA dressings (1 day).

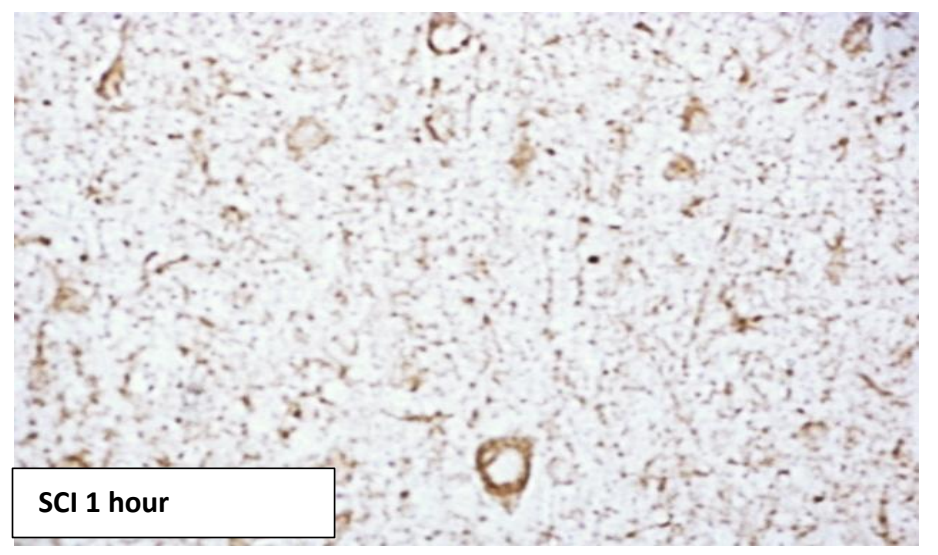



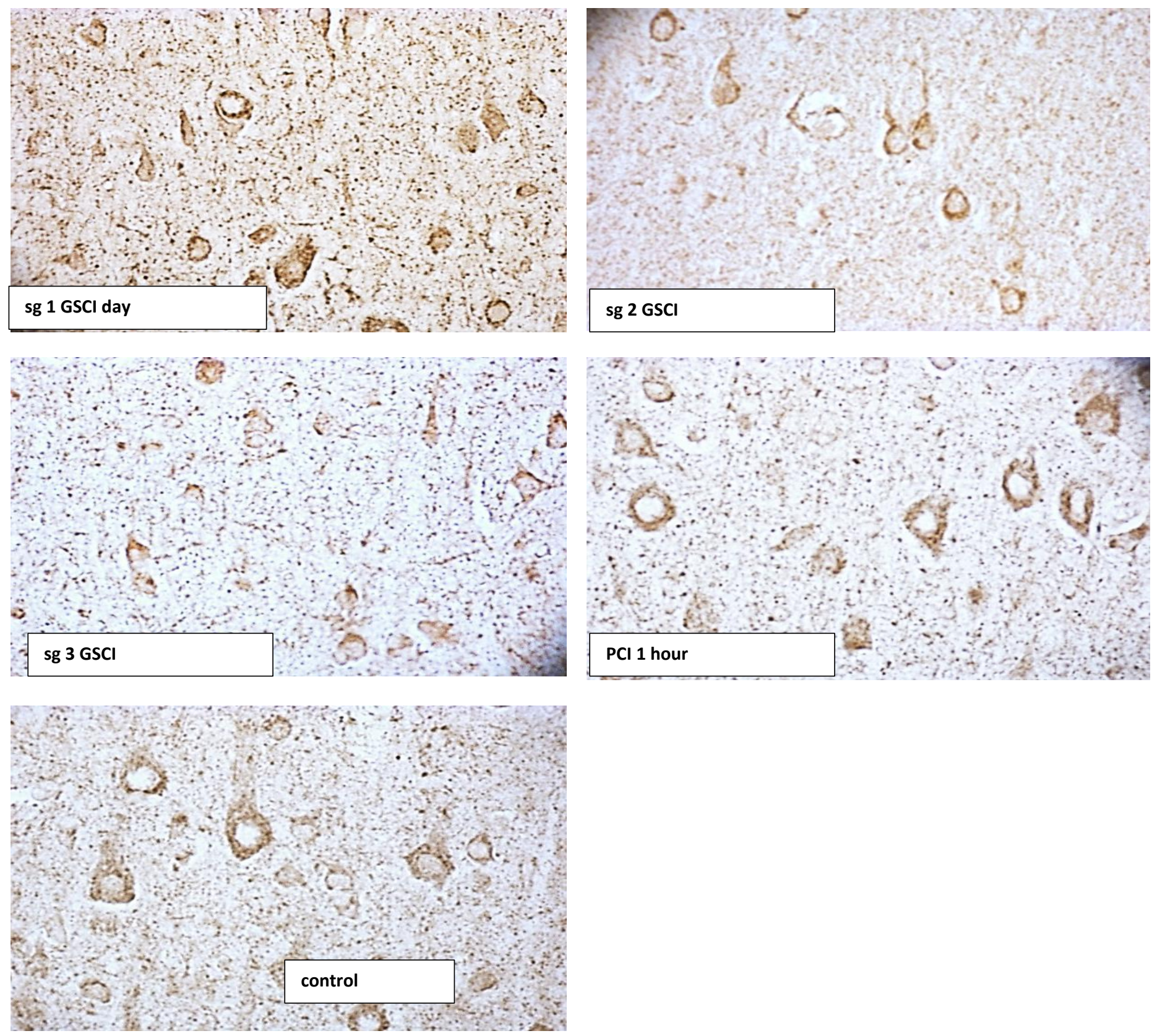

Note:

subgroup 1 - the interval between CCA dressings 7 days subgroup 2 - the interval between CCA dressings is 3 days subgroup 3 - the interval between CCA dressings 1 day

Figure 1. The content of ATP synthase in the cytoplasm of pyramidal neurons in the parietal cortex of rats with total (TCI), subtotal (SCI), stepwise subtotal (GSCI) and partial cerebral ischemia (PCI). Digital micrograph. Magnification $x 40$. 

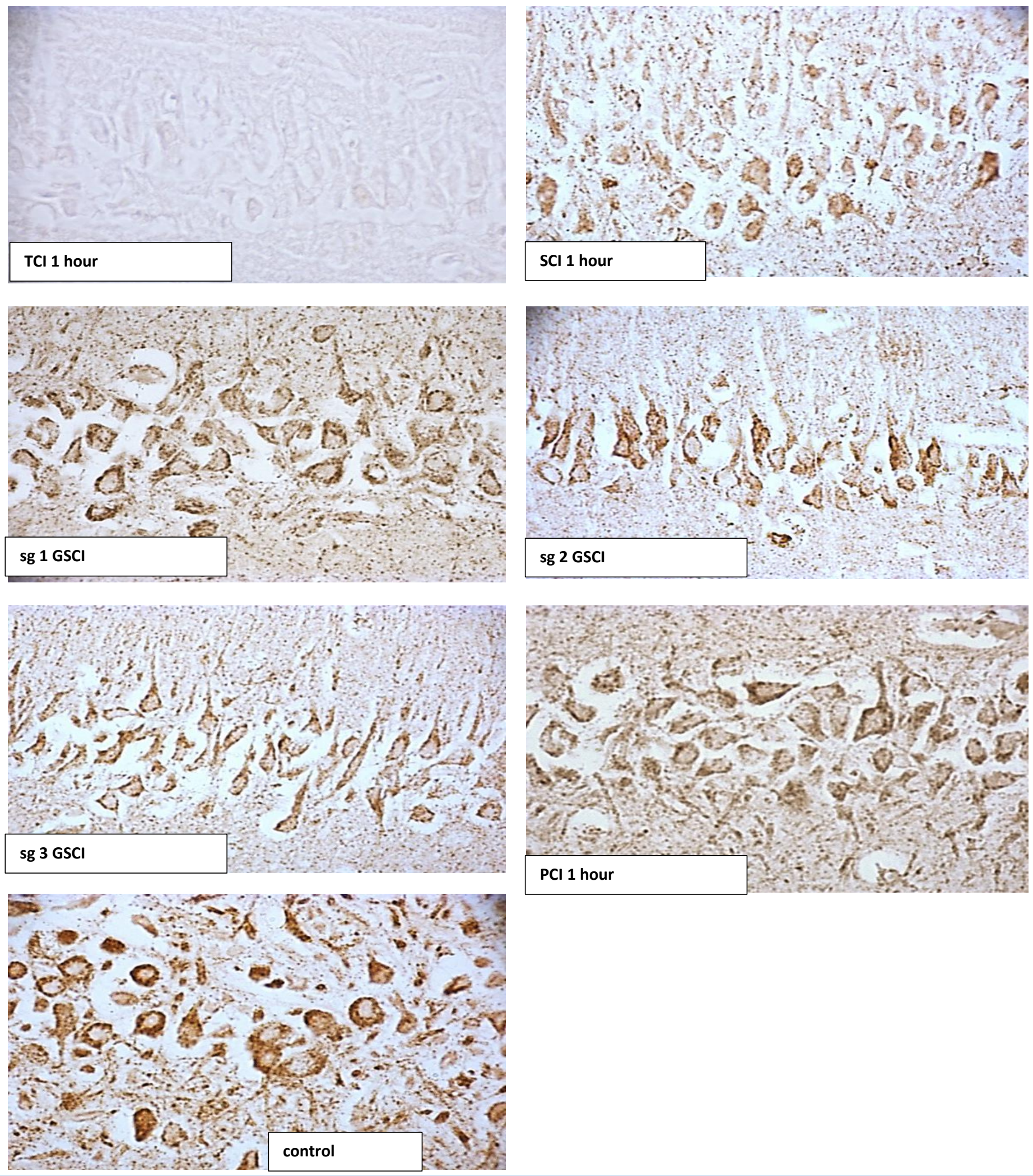

Figure 2. The content of ATP synthase in the cytoplasm of pyramidal neurons of the CA1 field of the hippocampus of rats with total (TCI), subtotal (SCI), stepwise subtotal (GSCI) and partial cerebral ischemia (PCI). Digital micrograph. Magnification $x 40$

Note:

subgroup 1 - the interval between CCA dressings 7 days

subgroup 2 - the interval between CCA dressings is 3 days

subgroup 3 - the interval between CCA dressings 1 day 


\section{Discussion}

Compared with the value in the "SCI" group, modeled by one-stage ligation of both CCA, in the 3rd subgroup of SCI in both studied departments, no differences were found ( $p>0.05)$. The content of ATP synthase in the 2 nd subgroup of SCI was $17 \%$ higher than at SCI $(p<0.05)$ in the hippocampus, while in the parietal cortex there were no differences (p>0.05), and in 1 -th subgroup SCI - it was $30 \%$ more in the parietal cortex $(\mathrm{p}<0.05)$ and $34 \%$ - in the hippocampus $(\mathrm{p}<0.05)$ than in rats with SCI.

Compared with the value in the group "PCI", in the 1st subgroup of GSCI in both studied departments there were no differences in the content of ATP synthase ( $p>0.05$ ), in the 2nd subgroup of GSCI it was less by $18 \%$ in the parietal cortex $(\mathrm{p}<0.05)$ and by $20 \%$ - in the hippocampus ( $\mathrm{p}$ $<0.05$ ), and in the 3rd subgroup of SIGM the differences from SCI were more pronounced and amounted to $26 \%$ ( $\mathrm{p}<0.05)$ and $27 \%(\mathrm{p}<0.05)$, respectively.

The severity of changes in the content of ATP-synthase in the 2nd and 3rd subgroups of GSCI was greater: by $18 \%$ ( $\mathrm{p}<0.05$ ) - in the hippocampus of the brain of rats of the second subgroup of SSHM and by $21 \%$ - in the parietal cortex $(\mathrm{p}<0,05)$ and $25 \%$ in the hippocampus $(\mathrm{p}<0.05)$ in the $3 \mathrm{rd}$ subgroup of rats with SCI.

The content of ATP-synthase in the 1st subgroup of SSIGM (the interval between CCA dressings was 7 days) was the closest to the indicators in the "PCI" group, while in the 3rd subgroup of GSCI, with a minimum interval between CCA dressings of 1 day, in to a greater extent was close to the values of the enzyme content in the "SCI" group, modeled by simultaneous ligation of both CCA, which indicates a more pronounced damage to the $\mathrm{V}$ complex of the electron transport chain in this model of SCI.

Studies have shown the dependence of the severity of brain damage in SSHM on the interval between the cessation of blood flow in both CCA. With a 7-day interval between CCA dressings, the content of ATP synthase was restored. The results obtained agree with the previously obtained data. According to which the smallest morphological changes in neurons were noted in the "CHIGM" groups and the 1st subgroup "GSCI", with an interval between CCA dressings of 7 days. It is obvious that with these methods of modeling IHM, adaptation processes occur that prevent the development of pronounced morphological changes and allow neurons to adapt to conditions of moderate hypoxia. So, for example, in PCI, the absence of pronounced morphological changes in rats is explained by the compensation of blood circulation in the circle of Willis. With GSCI, when the time interval (7 days between dressings) is sufficient for the development of adaptive processes, the productivity of mitochondrial respiration increases [5], and, possibly, the production of nitrogen monoxide and hypoxia-induced factor are activated [4]. Modeling of more severe types of ischemic damage leads to pronounced morphological changes in neurons in the parietal cortex and hippocampus of the rat brain - a decrease in their size, deformation of perikarya, an increase in the degree of neuronal chromatophilia with their simultaneous wrinkling and subsequent death. To the greatest extent, these disorders were expressed in the 3rd subgroup of SCI with the shortest interval between dressings, which was 1 day, and in the TCI group [4].

This, probably, can explain, in comparison with the groups "TCI", "SCI" and the 3rd subgroup "SCI", the higher content of ATP synthase in the cytoplasm of neurons in the 1st and 2nd subgroups "SCI" and " PCI ".

\section{Conclusion}

Thus, the most pronounced decrease in the content of ATP synthase was observed in the groups "TCI", "SCI" and in the 3rd subgroup "SCI", with a minimum time interval between CCA dressings. With GSCI with an interval between CCA dressings of 7 days, the suppression of the content of ATP synthase was not so significant [20,21].

\section{Acknowledgments}

Conflict of interest statement

The authors declare no conflict of interest.

\section{Funding sources}

State scientific program «To study the processes of damage and adaptation of the brain during its ischemia and the use of correction».

\section{References}

1. Maksimovich N.Ye. (2019) Structural and functional features of mitochondria and methods of their study in experiment. MEDICUS. 5:8-18.

2. Chan, P.H. (2004) Mitochondria and neuronal death/survival signaling pathways in cerebral ischemia. Neurochemical Research. 29:1943-1949.

3. Bon, L.I. (2021) Histological disorders of neurons of phylogenetically different parts of the cerebral cortex in partial, subtotal, stepwise subtotal, and total cerebral ischemia. Journal of Medical Science. 1:108-115.

4. Bon, L.I. (2021) Evaluation of neurological deficiency in rats with cerebral ischemia following the administration of omega polyunsaturated fatty acids. Journal of Medical Science. 3:137-143.

5. LaManna, J. C. (1992) Brain adaptation to chronic hypobaric hypoxia in rats. Journal of Applied Physiology. 72: 2238-2243.

6. Patt S. (1997) Cerebral angiogenesis triggered by severe chronic hypoxia displays regional differences. Journal of Cerebral Blood Flow \& Metabolism. 17: 801-806.

7. Kwiatkowski S., Knap B., Przystupski D. (2018) Photodynamic therapy - mechanisms, photosensitizers and combinations. Journal of Biomedicine \& Pharmacotherapy. 106:1098-1107.

8. DePierre J.W., Ernster L. (1988) Enzyme topology of intracellular membranes. Review Biochemistry. 46: 201-261.

9. Boyer, P.D. (1998) ATP synthase - past and future. Biochimica et Biophysica Acta. 1365:3-9.

10. Brand M.D., Murphy M.P. (1987) Control of electron flux through the respiratory chain in mitochondria and cells. Biological Review. 62: 141-193.

11. Casey R.P. (1984) Membrane reconstruction of the energyconserving enzymes of oxidative phosphorylation. Biochemistry Acta. 768: 319-347.

12. Pecina P., Nuskova H., Karbanova V., Kaplanova V. (2018) Role of the mitochondrial ATP synthase central stalk subunits $\gamma$ and $\delta$ in the activity and assembly of the mammalian enzyme. Acta Bioenergetics. 5:374-381.

13. Prince R.C. (1988) The proton pump of cytochrome oxidase. Trends Biochemistry Science. 13:159-160.

14. Srere P.A. (1982) The structure of the mitochondrial inner membrane-matrix compartment. Trends Biochemistry Science. 7:375-378.

15. Teixeira F.K., Sanchez C.G., Hurd T.R., Seifert J.R., Czech B., et al. (2015) ATP synthase promotes germ cell differentiation independent of oxidative phosphorylation. Journal of Natural Cell Biology. 5:689-696. 
16. Flavia S., Siavash H. (2016) Identification of neutrophil surface marker changes in health. Journal of Experimental Cell Research.2:200-209.

17. Maksimovich N.Ye. (2019) Structural and functional features of mitochondria and methods of their study in experiment. MEDICUS. 5:8-18.

18. Zhang Y., Chen W. (2016) Protective effects of mangiferin on cerebral ischemia-reperfusion injury and its mechanisms. European Journal of Pharmacology. 771:145-151.
19. Bon, L. I. (2020) Morphology of rat brain neurons in subtotal ischaemia and introduction of L-NAME and omega-3 polyunsaturated fatty acids. Journal of Medical Science. 1:1-8.

20. Timir T., Krishnananda Ch. (2019) Interaction of $\alpha$-Synuclein with ATP Synthase: Switching Role from Physiological to Pathological. Journal of ACS Chemical Neuroscience. 1:16-17.

21. Manuela A., Valentina G., Federico F. (2014) The oligomycinsensitivity conferring protein of mitochondrial ATP synthase: emerging new roles in mitochondrial pathophysiology. International Journal of Molecular Sciences. 5:7513-7536.
This work is licensed under Creative Commons Attribution 4.0 License

To Submit Your Article Click Here: $\quad$ Submit Manuscript
Ready to submit your research? Choose Auctores and benefit from:

$>$ fast, convenient online submission

$>$ rigorous peer review by experienced research in your field

$>$ rapid publication on acceptance

$>$ authors retain copyrights

$>$ unique DOI for all articles

$>$ immediate, unrestricted online access

At Auctores, research is always in progress.

Learn more https://auctoresonline.org/journals/biotechnology-andbioprocessing- 